\title{
Nausea Visual Analogue Scale
}

National Cancer Institute

\section{Source}

National Cancer Institute. Nausea Visual Analogue Scale. NCI Thesaurus. Code C141559.

A nausea scale from 0 to 10 on which the patient marks the current level of nausea experienced. 